\title{
Blueberry anthocyanin-enriched extract ameliorates transverse aortic constriction-induced myocardial dysfunction via the DDAH1/ADMA/NO signaling pathway in mice
}

\author{
WEIQING HU ${ }^{1}$, WENYUE WANG ${ }^{2}$, QING MA ${ }^{3}$, TAO LIU ${ }^{1}$, JIEFENG ZHANG $^{1}$ and JICUN ZHANG ${ }^{1}$ \\ ${ }^{1}$ Department of Vascular Surgery, The People' Hospital of Weifang City, Weifang, \\ Shandong 261000; Departments of ${ }^{2}$ Obstetrics and Gynecology, and ${ }^{3}$ Pharmacy, \\ Weifang Hospital of Traditional Chinese Medicine, Weifang, Shandong 261041, P.R. China
}

Received January 10, 2019; Accepted September 27, 2019

DOI: $10.3892 / \mathrm{mmr} .2019 .10800$

\begin{abstract}
Blueberry anthocyanin-enriched extract (BAE) has been demonstrated to protect against cardiovascular diseases by activating multiple target genes. The present study investigated the effects of BAE on transverse aortic constriction (TAC)-induced myocardial dysfunction in mice and explored its possible molecular mechanisms. A total of 30 male mice were divided randomly into control, TAC and TAC + BAE groups. Mice in the TAC + BAE groups were administered BAE by oral gavage for 6 consecutive weeks. Myocardial dysfunction was assessed using echocardiogram, histopathology, TUNEL assay, immunofluorescence staining, reverse transcription-quantitative PCR and western blot analysis. The results demonstrated that BAE treatment significantly ameliorated heart weight, left ventricular weight, myocardial dysfunction, left ventricular hypertrophy and fibrosis. In addition, BAE treatment alleviated TAC-induced inflammation, oxidative stress and apoptosis. Notably, BAE treatment markedly reduced asymmetric dimethylarginine (ADMA) concentration and significantly increased dimethylarginine dimethylaminohydrolase 1 (DDAH1) expression and nitric oxide (NO) production. The present data indicated that BAE treatment ameliorated TAC-induced myocardial dysfunction, oxidative stress, inflammatory response and apoptosis via the DDAH1/ADMA/NO signaling pathway.
\end{abstract}

Correspondence to: Professor Jicun Zhang, Department of Vascular Surgery, The People' Hospital of Weifang City, 151 Guangwen Street, Kuiwen, Weifang, Shandong 261000, P.R. China

E-mail: jicunzhang8@126.com

Key words: blueberry anthocyanin-enriched extract, transverse aortic constriction, myocardial dysfunction, oxidative stress, inflammation, dimethylarginine dimethylaminohydrolase 1 , asymmetric dimethylarginine, nitric oxide

\section{Introduction}

Transverse aortic constriction (TAC) causes compensatory hypertrophy, chronic maladaptive hemodynamic overload, cardiac dilatation and heart failure over time (1). TAC is often used as an experimental method to produce pressure overload, cardiac hypertrophy and heart failure in mice (2). Although it has been well demonstrated that TAC-induced myocardial dysfunction is associated with inflammation, oxidative stress and cardiomyocyte apoptosis (3-6), the molecular signaling pathways involved in TAC-induced myocardial dysfunction have yet to be elucidated. Therefore, it is important to investigate possible target genes and to identify possible treatments for TAC-induced myocardial dysfunction.

Asymmetric dimethylarginine (ADMA) is reported to be an endogenous nitric oxide synthase (NOS) inhibitor, and its accumulation is associated with various cardiovascular diseases, including hypertension, diabetes and cardiac dysfunction (7-10). ADMA attenuates endothelial NOS (eNOS) activity to reduce nitric oxide (NO) production and induce NOS uncoupling to generate reactive oxygen species (ROS) $(8,11)$. Dimethylarginine dimethylaminohydrolase 1 (DDAH1) serves an important role in regulating vascular endothelial injury repair and angiogenesis, as well as functions in degrading ADMA to maintain NO signaling $(12,13)$. Overexpression of the DDAH1 gene reduces inflammatory infiltration through decreasing ADMA expression (14). Serum ADMA concentration was slightly higher when left ventricle DDAH1 expression was low, while DDAH1 knock out (KO) exacerbated left ventricle hypertrophy, fibrosis and dysfunction in mice after TAC (15). These findings suggest that DDAH1 may protect cardiac hypertrophy and ventricular remodeling against stress conditions.

Berry anthocyanins have recently drawn widespread scientific interest due to their diverse health benefits, including antioxidant, anti-inflammatory, antihypertensive, anti-atherosclerotic, antimicrobial, anticancer and neuroprotective properties (16). According to National Health and Nutrition Education Survey (NHANES), an average of $12.5 \mathrm{mg}$ of anthocyanins are consumed per day by people in the United States (17). In vitro and in vivo studies have shown that blueberry 
anthocyanins activate cellular antioxidant systems and inhibit inflammatory infiltration, and protect against inflammation and oxidative stress, through activation of multiple target genes. The present study investigated the effects and potential mechanisms of blueberry anthocyanin-enriched extract (BAE) on TAC-induced myocardial dysfunction.

\section{Materials and methods}

Experimental animals. A total of 30 male C57BL/6 mice (age, 6-8 weeks; weight, 20-25 g) were obtained from the Experimental Animal Center of the General Hospital of Shenyang Military Command. All animals were housed at $20 \pm 2^{\circ} \mathrm{C}$ and $55-60 \%$ humidity under a 12 -h light/dark cycle, were provided standard laboratory animal feed and water ad libitum, and were allowed to acclimate for 7 days before the study. All experimental animal procedures were approved by the Animal Ethics Committee of the People's Hospital of Weifang City.

Materials and reagents. BAE was provided by the Food College of Shenyang Agricultural University (18). The total anthocyanin content was $\sim 25.7 / 100$ g of extract. The composition of BAE, as measured by high-performance liquid chromatography/mass spectrometry, was: Malvidin 3-galactoside $(28.11 \%)$, malvidin 3 -arabinoside (16.18\%), malvidin 3 -glucoside (14.08\%), malvidin 3-(6"-acetyl) glucoside $(8.49 \%)$, malvidin 3-(6"-acetyl) galactoside $(5.50 \%)$, petunidin 3 -galactoside $(5.44 \%)$, petunidin 3 -glucoside $(5.26 \%)$, peonidin3-glucoside $(5.22 \%)$, cyanidin3-galactoside $(2.96 \%)$ and delphinidin 3-glucoside (1.41\%).

Experimental protocol. After acclimation, the 30 mice were divided randomly into control, TAC and BAE groups. The mice in the control group did not undergo surgery. The mice in the BAE group were modelled for TAC and, following a recovery period of $24 \mathrm{~h}$ after the surgery, they were administered with $0.5 \mathrm{~g} / \mathrm{kg}$ BAE daily by oral gavage for 6 consecutive weeks. Mice in the control and TAC groups were given the same volume of distilled water.

TAC model. TAC models were created as described previously (19). Briefly, mice were anesthetized in an induction chamber with $2 \%$ isoflurane mixed with $0.5-1.0 \mathrm{l} / \mathrm{min} 100 \%$ $\mathrm{O}_{2}$. Mice were fixed in a supine position on top of a heating pad to maintain body temperature. Partial thoracotomy at the second rib was performed under a surgical microscope and the sternum was retracted using a chest retractor. Fine tip $45^{\circ}$ angled forceps were used to gently separate the thymus and fat tissue from the aortic arch. Following identification of the transverse aorta, a small piece of a 6.0 silk suture was placed between the innominate and left carotid arteries. Two loose knots were tied around the transverse aorta and a small piece of a gauge blunt needle was placed parallel to the transverse aorta. The first knot was quickly tied against the needle, followed by the second one, and the needle was promptly removed to yield a constriction of $0.4 \mathrm{~mm}$ in diameter. The chest retractor was removed and the outflow of the ventilator pinched off for $2 \mathrm{sec}$ to re-inflate the lungs. The rib cage was closed using a 6.0 prolene suture with an interrupted suture pattern. The skin was closed using a 6.0 prolene suture with a continuous suture pattern.

Echocardiogram. All animals were anesthetized with $1.6 \%$ isoflurane and were assessed by echocardiogram (ECHO; Vevo 770, a $12 \mathrm{MHz}$ transducer; VisualSonics Inc.) according to previous literature (20). Briefly, to ensure that the mitral and aortic valves and the apex were visualized, parasternal long axis views were obtained and recorded. Short axis views were recorded at the mid-papillary muscle level. To calculate the end-systolic left ventricular (LV) area, endocardial area tracings were obtained using 2D mode from digital images captured on cine-loop. All measurements were made by a single observer and were averaged over 3-5 consecutive cardiac cycles. The reproducibility of measurements was assessed in two sets of baseline measurements in 10 randomly selected rats. Repeated measure variability did not exceed $65 \%$.

Sample collection. Briefly, mice were anesthetized by intraperitoneal injection of $2 \%$ pentobarbital sodium $50 \mathrm{mg} / \mathrm{kg}$ of body weight (1.5 ml/kg; cat. no. 57-33-0; year 2016; Shanghai Haohai Biological Technology Co., Ltd.) and then sacrificed. Tissues and blood samples were obtained from abdominal aortas and stored for analysis. Heart and lung tissues were also collected. Following weighing on an electronic balance, tissues were either fixed in $10 \%$ formaldehyde at room temperature for 3-5 days or immediately frozen in liquid nitrogen and then transferred to a $-80^{\circ} \mathrm{C}$ freezer.

ELISA. Levels of inflammatory factors, including interleukin (IL)-1 $\beta$ and tumor necrosis factor (TNF)- $\alpha$ were measured with ELISA kits (cat. nos. ab197742 and ab208348, respectively; Abcam). The concentrations of the heart ADMA (cat. no. CEB301Ge, Cloud-Clone Corp.) and NO (cat. no. BH3702; Shanghai Kaibo Biology Technology Co., Ltd.) were measured with ELISA kits, according to the manufacturers' directions. The absorbance was measured at $450 \mathrm{~nm}$ using an Enzyme Labeled Instrument (Bio-Rad Laboratories, Inc.), and the concentration of NO and ADMA in the samples was calculated by standard curve.

Histopathological analysis. Samples for histological analysis were immersed in $10 \%$ formalin buffer at room temperature for 3-5 days, embedded in paraffin using a Leica Microsystem tissue processor (ASP 300S), and sliced into 3-4 $\mu \mathrm{m}$ sections using a Leica Microsystem microtome (model RM 226; Leica Microsystems GmbH). These sections were stained with hematoxylin and eosin (H\&E) at room temperature for $10 \mathrm{~min}$. LV fibrosis was estimated by staining with modified Masson's Trichrome Stain kit (Sigma-Aldrich; Merck KGaA).

Reverse transcription-quantitative $(R T-q) P C R$. Total RNA was extracted from heart tissue with TRIzol (Takara Bio, Inc.) and reverse-transcribed using the SuperScript ${ }^{\mathrm{TM}}$ Double-Stranded cDNA Synthesis kit (Invitrogen; Thermo Fisher Scientific, Inc.), following the suppliers' protocols. Reactions were performed in an RT-qPCR thermocycler (Bio-Rad Laboratories, Inc.) using SYBR Green PCR Master Mix (Takara Bio Inc.). The thermocycling conditions were: $94^{\circ} \mathrm{C}$ for $5 \mathrm{~min}$, followed by 
35 cycles of $94^{\circ} \mathrm{C}$ for $35 \mathrm{sec}$ (denaturation), $57.3^{\circ} \mathrm{C}$ for $35 \mathrm{sec}$ (annealing), and $72^{\circ} \mathrm{C}$ for $50 \mathrm{sec}$ (extension). Primer sequences were: Atrial natriuretic peptide (ANP), forward 5'-AGCGAG CAGACCGATGAAG-3' and reverse 5'-AGCCCTCAGTTT GCTTTTCA-3'; brain natriuretic peptide (BNP), forward 5'-TGATTCTGCTCCTGCTTTC-3' and reverse 5'-GTGGAT TGTTCTGGAGACTG-3'; myosin heavy chain $\beta$ ( $\beta$-MHC), forward 5'-AGAGCAAAAGCAAAGGGTTTC-3' and reverse 5'-GTGATGGTACGAGATGGGCTA-3'; IL-1 $\beta$, forward 5'-CCTGTTCTTTGAAGTTGACGG-3' and reverse 5'-AGC TTCTCCACAGCCACAAT-3'; TNF- $\alpha$, forward 5'-CCACCA CGCTCTTCTGTCTA-3' and reverse 5'-GAGAGGGAGGCC ATTTGGGA-3'; DDAH1, forward 5'-AGGTGCTGAAAT CTTGGCTG-3' and reverse 5'-GCAGATTCGCTGGACCCT AT-3'; melanoma differentiation-associated protein 5 (MDA5), forward 5'-AGTGTCTCCACTTGCTGACC-3' and reverse 5'-CAGCAGCTCTCTTACACCTGA-3'; inositol-requiring enzyme $\alpha$ (IRE $\alpha$ ), forward 5'-AGCACAGTTACACTGCCT GAG-3' and reverse 5'-CTTCCACGTGTGTTGGGACCT-3'; superoxide dismutase-1 (SOD-1), forward 5'-GAGCATTCC ATCATTGGCCG-3' and reverse 5'-GGCAATCCCAATCAC ACCAC-3'; Bax, forward 5'-TCCACCAAGAAGCTGAGC GA-3' and reverse 5'-TTGAAGTTGCCATCAGCAAACA-3'; Caspase-3, forward 5'-ATGGGAGCAAGTCAGTGGAC-3' and reverse 5'-GTCCACATCCGTACCAGAGC-3'; Bcl-2, forward 5'-TGAGTACCTGAACCGGCATC-3' and reverse 5'-AAGCCCAGACTCATTCAACCA-3'; Caspase-8, forward 5'-CCTAGACTGCAACCGAGAGG-3' and reverse 5'-TCC AACTCGCTCACTTCTTCTG-3'; and GAPDH, forward 5'-CGGATTTGGTCGTATTGGG-3' and reverse 5'-CTG GAAGATGGTGATGGGATT-3'. The mRNA expression of the target genes was normalized to GAPDH as an internal control, and relative fold changes in mRNA expression were calculated using the formula $2^{-\Delta \Delta \mathrm{Cq}}(21)$.

Western blot analysis. Western blotting was performed as previously described (22). Briefly, heart tissues were lysed in radioimmunoprecipitation assay (RIPA) buffer $(10 \mathrm{mM}$ Tris- $\mathrm{HCl} \mathrm{pH} 7.4,150 \mathrm{mM} \mathrm{NaCl}, 1 \% \mathrm{NP} 40,0.1 \%$ sodium dodecyl sulfate, $1 \mathrm{mM}$ phenylmethylsulfonyl fluoride and $1 \mathrm{X}$ protease inhibitor cocktail; Roche Diagnostics) and homogenized by Sonic Dismembrator 100 (Thermo Fisher Scientific, Inc.). The protein concentration of tissue homogenates was measured using a Bio-Rad Protein Assay (Bio-Rad Laboratories, Inc.). A total of $30 \mu \mathrm{g}$ soluble protein was separated on $10 \%$ polyacrylamide gels and transferred into nitrocellulose membrane. The membrane was blocked with $5 \%$ skimmed milk powder at room temperature for $1 \mathrm{~h}$. The following primary antibodies were used: TNF- $\alpha$ (1:2,000; ab8348; Abcam), IL-6 (1:1,000; ab83053; Abcam), IRE $\alpha$ (1:2,000; 3294; Abcam), MDA5 (1:200; ab69983; Abcam), ANP (1:500; ab251006; Abcam), BNP (1:1,000; ab239510; Abcam), $\beta$-MHC (1:200; ab23990; Abcam), SOD-1 (1:1,000; sc-11407; Santa Cruz Biotechnology, Inc.), Bax (1:2,000; sc-526; Santa Cruz Biotechnology, Inc.), Caspase 3 (1:500; sc-7148; Santa Cruz Biotechnology, Inc.), Bcl-2 (1:1,000; sc-7382; Santa Cruz Biotechnology, Inc.), Caspase 8 (1:100; sc-5263; Santa Cruz Biotechnology, Inc.), DDAH1 (1:500; sc-5268; Santa Cruz Biotechnology, Inc.) and GAPDH (1:5,000; sc-32233; Santa Cruz Biotechnology, Inc.). The secondary
Table I. Cardiac function parameters in the experimental groups.

\begin{tabular}{lrrr}
\hline Parameters & Control $(\mathrm{n}=10)$ & TAC $(\mathrm{n}=10)$ & BAE $(\mathrm{n}=10)$ \\
\hline LVPW $(\mathrm{mm})$ & $2.39 \pm 0.36$ & $12.01 \pm 0.27^{\mathrm{a}}$ & $5.71 \pm 0.38^{\mathrm{b}}$ \\
LVEDd $(\mathrm{mm})$ & $3.25 \pm 0.53$ & $4.91 \pm 0.47^{\mathrm{a}}$ & $3.91 \pm 0.32^{\mathrm{b}}$ \\
LVESd $(\mathrm{mm})$ & $1.72 \pm 0.47$ & $3.75 \pm 0.71^{\mathrm{a}}$ & $2.28 \pm 0.53^{\mathrm{b}}$ \\
LVEDV $(\mu \mathrm{l})$ & $47.25 \pm 6.04$ & $89.54 \pm 9.53^{\mathrm{a}}$ & $68.45 \pm 4.53^{\mathrm{b}}$ \\
LVESV $(\mu \mathrm{l})$ & $18.05 \pm 7.31$ & $55.34 \pm 6.93^{\mathrm{a}}$ & $31.13 \pm 2.05^{\mathrm{b}}$ \\
LVEF $(\%)$ & $72.32 \pm 5.95$ & $31.42 \pm 3.18^{\mathrm{a}}$ & $51.34 \pm 0.34^{\mathrm{b}}$ \\
LVFS $(\%)$ & $42.64 \pm 4.42$ & $23.65 \pm 3.08^{\mathrm{a}}$ & $32.32 \pm 6.38^{\mathrm{b}}$
\end{tabular}

Data are presented as mean \pm standard error of the mean. ${ }^{\mathrm{a}} \mathrm{P}<0.05$ vs. control group; ${ }^{b} \mathrm{P}<0.05$ vs. TAC group. TAC, transverse aortic constriction; BAE, blueberry anthocyanin-enriched extract; LV, left ventricle; LVPW, LV wall thickness; LVEDd, LV end-diastolic diameter; LVESd, LV end-systolic diameter; LVEDV, LV end-diastolic volume; LVEF, LV ejection fraction; LVFS, LV fractional shorting.

antibodies were: Horseradish peroxidase (HRP)-labeled goat anti-mouse secondary antibody (ab6789; 1:4,000; Abcam), goat anti-rabbit HRP-labeled secondary antibody (ab6721; 1:4,000; Abcam) and goat anti-rat HRP-labeled secondary antibody (ab7097; 1:2,000; Abcam). Secondary antibodies were incubated for $1.5 \mathrm{~h}$ at room temperature. Proteins were visualized using a ClarityTM Western ECL Substrate (cat. no. 170-5061; Bio-Rad Laboratories, Inc.) and a Tanon 5200 full automatic chemiluminescence image analysis system (Tanon Science and Technology Co., Ltd.).

Statistical analysis. Statistics were performed using SPSS 20.0 statistical software (IBM Corp.). Data were expressed as means \pm standard error of the mean and were analyzed using unpaired t-test and one-way ANOVA followed by a Bonferroni correction post hoc test for differences among $>2$ groups. $\mathrm{P}<0.05$ was considered to indicate a statistically significant difference.

\section{Results}

BAE ameliorates TAC-induced cardiac hypertrophy in mice. Compared with the control group, the TAC group had significantly higher heart weight and LV weight/heart weight ratio, but these effects were significantly attenuated by BAE treatment (Fig. $1 \mathrm{~A}$ and $\mathrm{B} ; \mathrm{P}<0.05$ ). Additionally, compared with the control group, the TAC group had significantly increased protein and mRNA expression levels of ANP, BNP and $\beta$-MHC in their heart LV tissues, and these were significantly ameliorated following BAE treatment (Fig. 1C-I; P<0.05).

BAE ameliorates TAC-induced LV dysfunction in mice. Compared with the control group, the TAC group demonstrated reduced LV fractional shorting and LV ejection fraction, and increased LV end-systolic diameter, LV end-diastolic diameter, and LV wall thickness. BAE treatment markedly elevated the LV ejection fraction and LV fractional shorting and decreased the LV end-diastolic and end-systolic diameters compared with the TAC group (Table I; $\mathrm{P}<0.05$ ). 

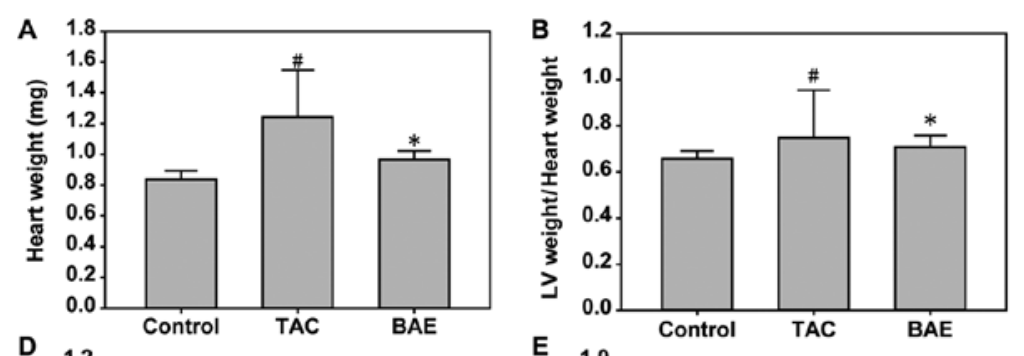

C
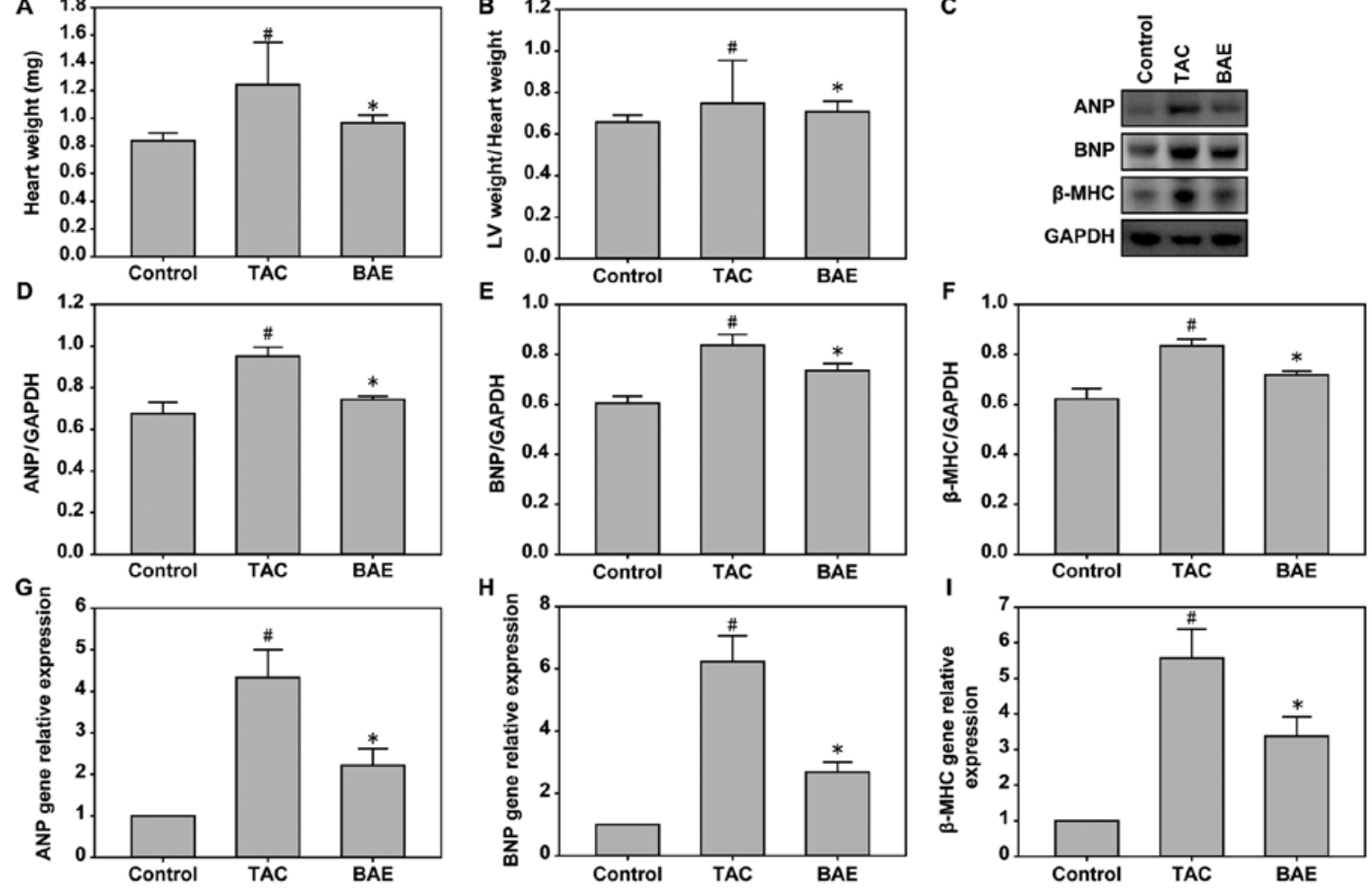

Figure 1. BAE ameliorates TAC-induced cardiac hypertrophy. (A) Heart weight (mg). (B) LV weight/heart weight ratio. (C) Representative western blot images and quantitative analysis of (D) ANP, (E) BNP and (F) $\beta$-MHC, with GAPDH used as loading control. (G) Reverse transcription-quantitative PCR analysis of ANP, (H) BNP and (I) $\beta$-MHC. All experiments were repeated at least three times. Data are presented as mean \pm standard error of the mean. ${ }^{\# P}<0.05 \mathrm{vs}$. control group; " $\mathrm{P}<0.05$ vs. TAC group. BAE, blueberry anthocyanin-enriched extract; TAC, transverse aortic constriction; LV, left ventricle; ANP, atrial natriuretic peptide; BNP, brain natriuretic peptide; $\beta$-MHC, myosin heavy chain $\beta$.

BAE ameliorates TAC-induced LV inflammation and fibrosis in mice. Compared with the control group, the TAC group experienced heart hypertrophy, edema, inflammatory cell infiltration, as well as a significant increase in the levels of pro-inflammatory cytokines IL-1 $\beta$ and TNF- $\alpha$ (Fig. 2). BAE treatment significantly reversed the TAC-induced pro-inflammatory factor secretion (Fig. 2; $\mathrm{P}<0.05$ ). In addition, TAC induced significant LV fibrosis compared with the control group, and this effect was ameliorated following BAE treatment (Fig. 2; $\mathrm{P}<0.05$ ).

$B A E$ ameliorates TAC-induced oxidative stress in mice. To assess the effect of BAE on TAC-induced oxidative stress, the protein and mRNA expression levels of MDA5 and IRE $\alpha$ (markers of oxidative stress), and of SOD-1 (antioxidant status marker), were assessed in the heart tissues of the experimental mice. The data demonstrated that TAC significantly increased MDA5 and IRE $\alpha$ expression, but decreased SOD-1 expression. By contrast, BAE treatment enhanced the expression of MDA5 and IRE $\alpha$ and reduced the expression of SOD-1 (Fig. 3; P<0.05).

BAE ameliorates TAC-induced heart tissue apoptosis in mice. The present data demonstrated that TAC significantly increased Bax and Caspase-3 expression, but decreased Bcl-2 and Caspase-8 expression, compared with the control group, at both the mRNA and protein levels. By contrast, BAE treatment significantly reversed the upregulation of Bax and Caspase-3 and the downregulation of Bcl-2 and Caspase- 8 induced by TAC (Fig. 4; $\mathrm{P}<0.05$ ).
BAE ameliorates TAC-induced myocardial dysfunction via DDAHI/ADMA/NO signaling in mice. To investigate whether DDAH1/ADMA/NO signaling was involved in TAC-induced myocardial dysfunction, the protein expression levels of DDAH1 and the concentrations of ADMA and NO were measured in heart tissues by western blot analysis, RT-qPCR and ELISA. Compared with the control group, TAC significantly increased ADMA concentration (Fig. 5E; P<0.05), but decreased DDAH1 expression (Fig. 5A-C; $\mathrm{P}<0.05$ ) and NO production (Fig. 5D; $\mathrm{P}<0.05$ ). Notably, BAE treatment significantly reversed these effects (Fig. 5; $\mathrm{P}<0.05$ ).

\section{Discussion}

Cardiovascular risk factors, including diabetes, smoking, hypertension, dyslipidemia, aging, and obesity, are associated with ROS, oxidative stress, inflammatory response and apoptosis (23). TAC increases free oxygen radicals, inflammation and apoptosis in heart tissues. The present study demonstrated that BAE ameliorated cardiac dysfunction, LV hypertrophy and fibrosis induced by TAC. The present data also demonstrated that BAE treatment attenuated TAC-induced LV leukocyte infiltration, inflammatory cytokine expression, oxidative stress and apoptosis. Furthermore, BAE significantly increased DDAH1 expression and NO production, and decreased ADMA concentration. These results suggested that TAC-induced myocardial dysfunction may be ameliorated by BAE treatment via the DDAH1/ADMA/NO signaling pathway. 
A

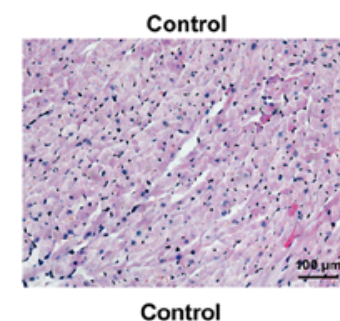

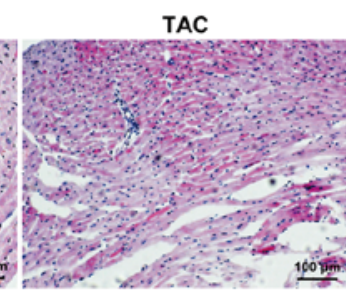

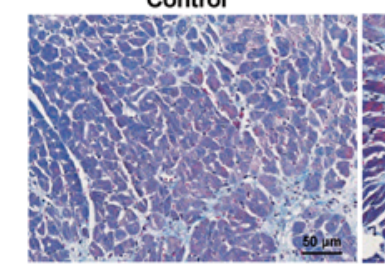

E
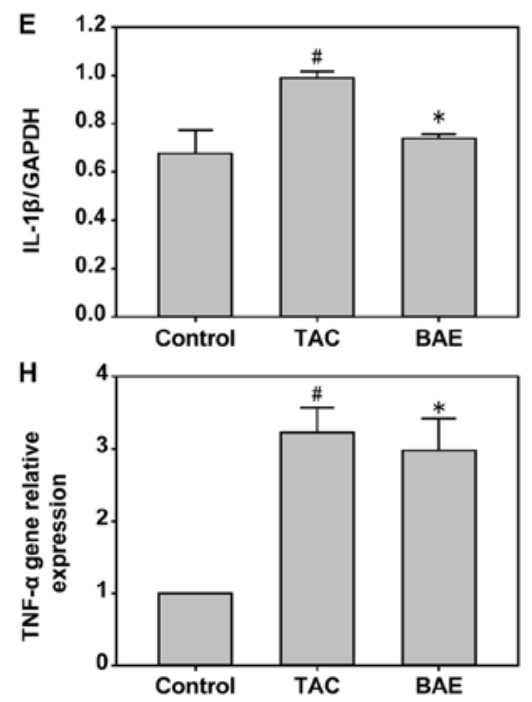

TAC
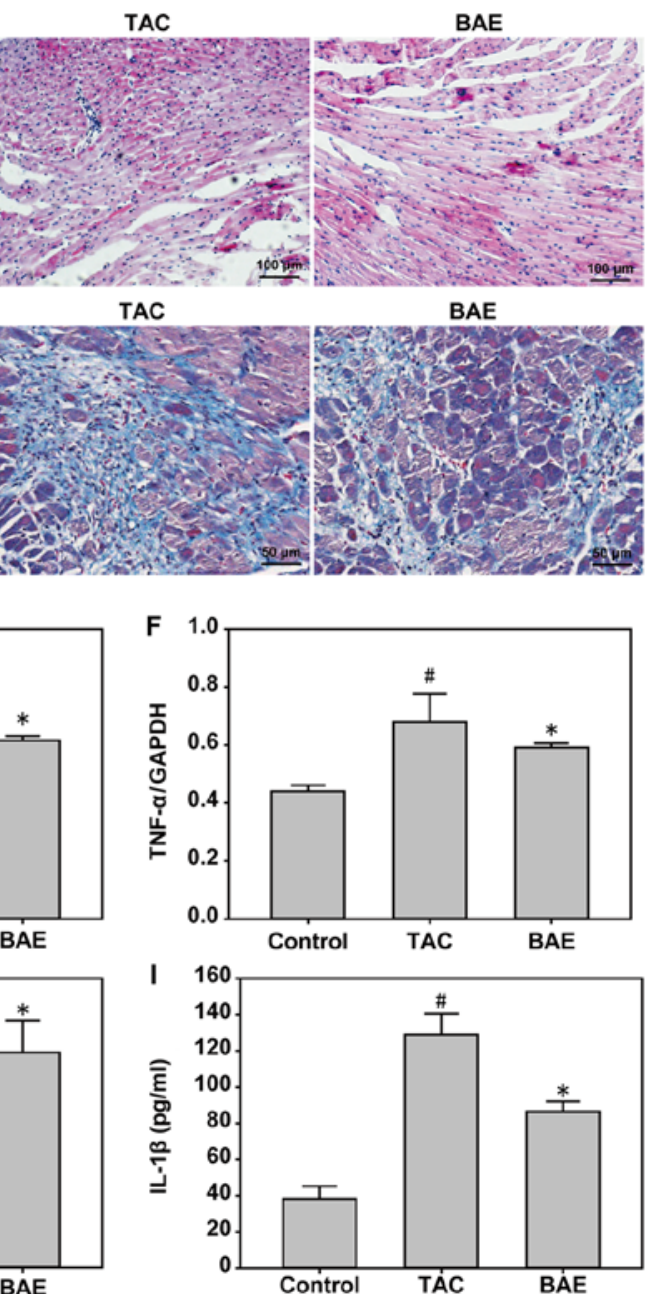

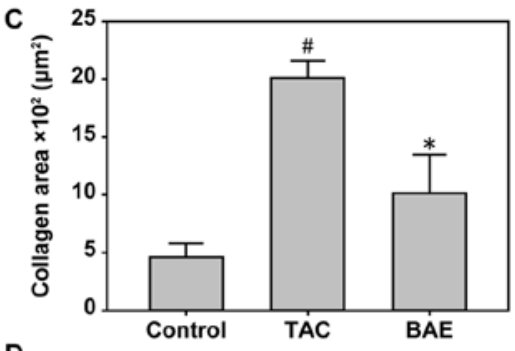

D
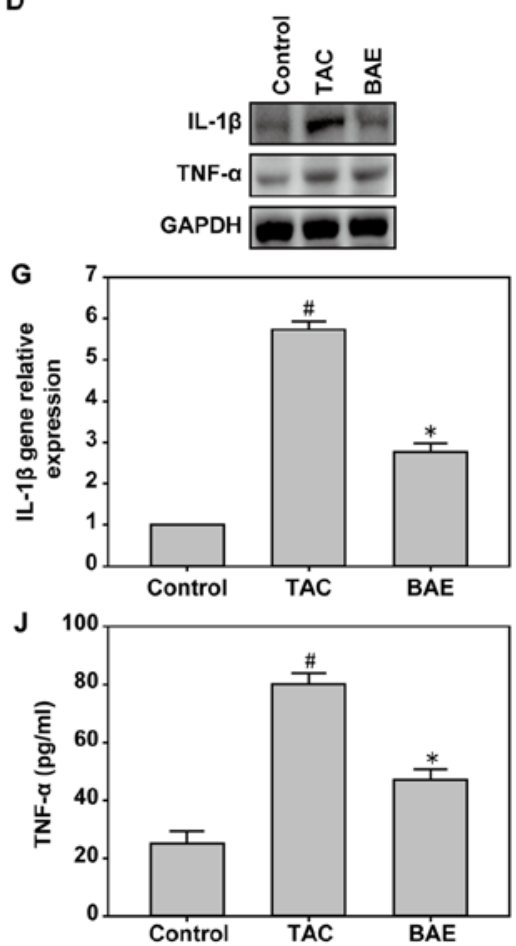

Figure 2. BAE ameliorates TAC-induced LV inflammation and fibrosis in mice. (A) H\&E and (B) Masson trichrome-stained sections of LV tissues. (C) Quantification of cardiac fibrosis area from Masson trichrome-stained sections. (D-F) Levels of inflammatory factors IL-1 $\beta$ and TNF- $\alpha$ were measured by western blotting, $(\mathrm{G}$ and $\mathrm{H})$ reverse transcription-quantitative PCR and (I and J) ELISA. All experiments were repeated at least three times. Data are presented as mean \pm standard error of the mean. ${ }^{*} \mathrm{P}<0.05$ vs. control; ${ }^{*} \mathrm{P}<0.05$ vs. TAC group. BAE, blueberry anthocyanin-enriched extract; $\mathrm{TAC}$, transverse aortic constriction; LV, left ventricle; IL, interleukin; TNF, tumor necrosis factor.

A previous study demonstrated that 20 and $80 \mathrm{mg} / \mathrm{kg} \mathrm{BAE}$ treatment attenuated cyclophosphamide-induced cardiac dysfunction, left ventricular hypertrophy and fibrosis (24). In addition, BAE significantly reduced systolic blood pressure and increased aortic vessel relaxation in response to acetylcholine in high fat/high cholesterol diet-fed rats $(25,26)$. Oxidative stress activates several intracellular signaling pathways and upregulates the expression of a large number of pro-inflammatory cytokines (27). A previous study demonstrated that a long-term blueberry-enriched diet decreases blood pressure and attenuates oxidative status in the kidneys of spontaneously hypertensive rats (28). BAE significantly inhibits hydrogen peroxide-induced human retinal pigment epithelial cell apoptosis, decreased vascular endothelial growth factor levels and activates protein kinase B (Act)-signal pathways (29). Song et al (30) demonstrated that BAE protects retinal cells against diabetes-induced oxidative stress and inflammation. In addition to the antioxidant effects, a previous study demonstrated that blueberry supplementation decreases serum inflammatory markers, including TNF- $\alpha$, IL-6 and C-reactive protein (31). Freeze-dried blueberries markedly reduce pro-inflammatory cytokines $\mathrm{TNF}-\alpha$ and IL-6 production in macrophages of apolipoprotein $\mathrm{E} \mathrm{KO}$ mice by inhibiting nuclear factor $(\mathrm{NF})-\kappa \mathrm{B}$ activation and the mitogen-activated protein kinase pathway (32). BAE attenuates $\mathrm{C}-\mathrm{C}$ motif chemokine ligand 4 -induced liver fibrosis, associated with reducing sources of ROS generation and associated oxidative damage, decreasing the influence of pro-inflammatory cytokines (33). Blackberry and blueberry anthocyanin supplementation significantly reduces serum and hepatic lipid levels, markedly elevates hepatic SOD and glutathione peroxidase activities, and reduces the expression of TNF- $\alpha$, IL-6 and NF- $\kappa$ B genes in high-fat diet fed C57BL/6 mice (34). Additionally, blueberry suppresses cell cycle progression and induces mitochondrial-mediated cell apoptosis by abolishing the Janus kinase/STAT3 pathway (35). In brief, the present data, in agreement with the previous literature, suggested that BAE may have a protective effect against TAC-induced LV inflammation, oxidative stress and apoptosis.

In vivo and in vitro experiments have demonstrated that the DDAH1/ADMA/NO signaling pathway is closely 
A
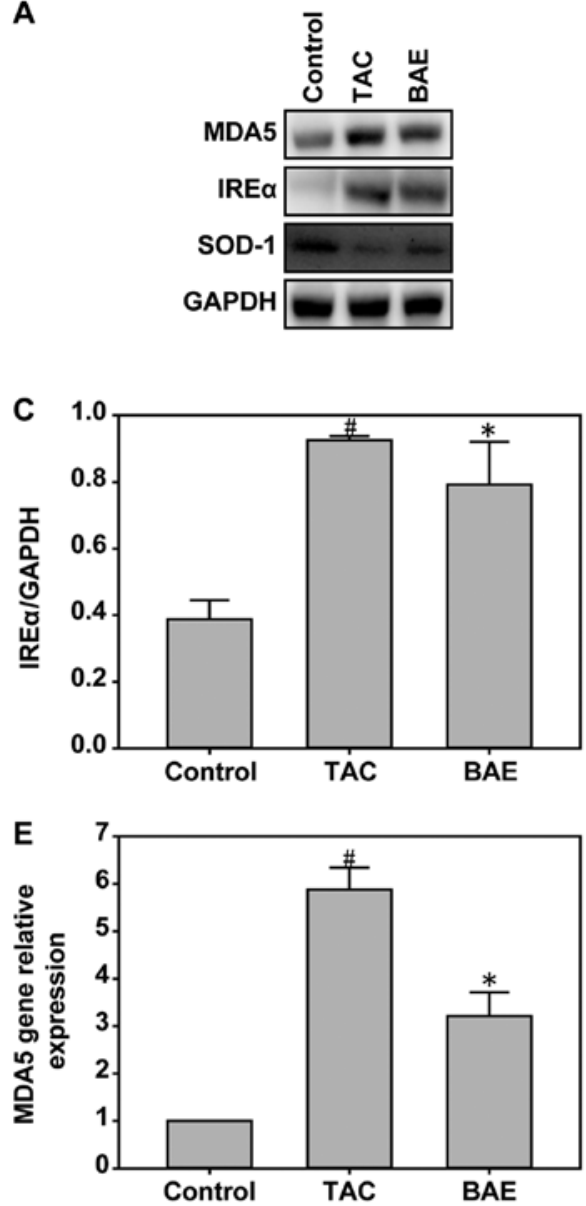
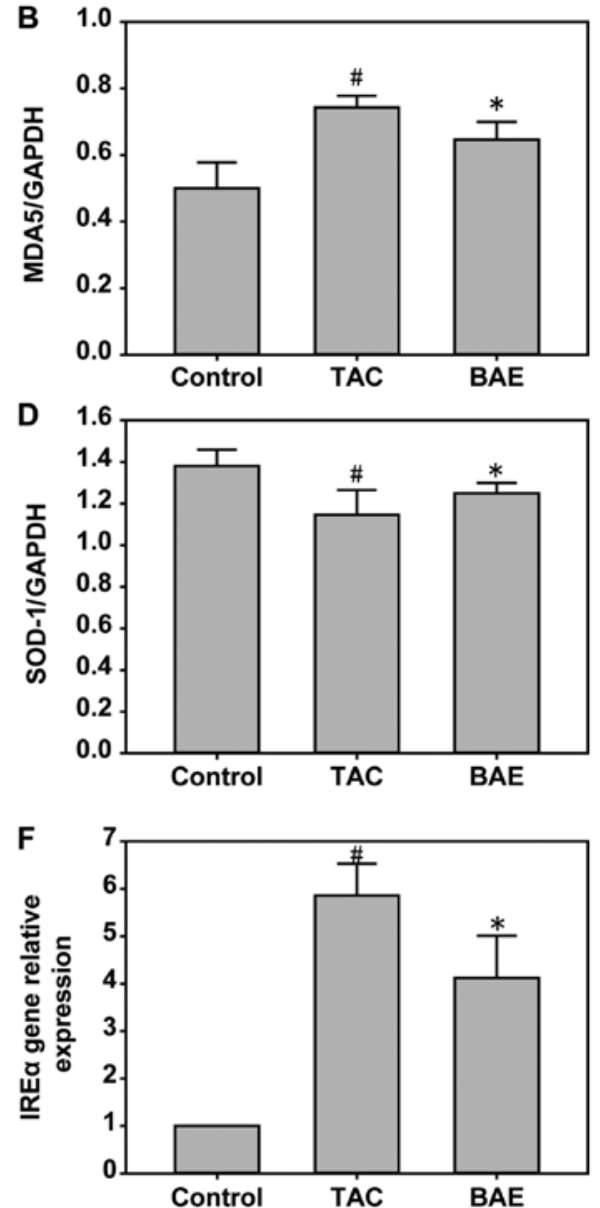

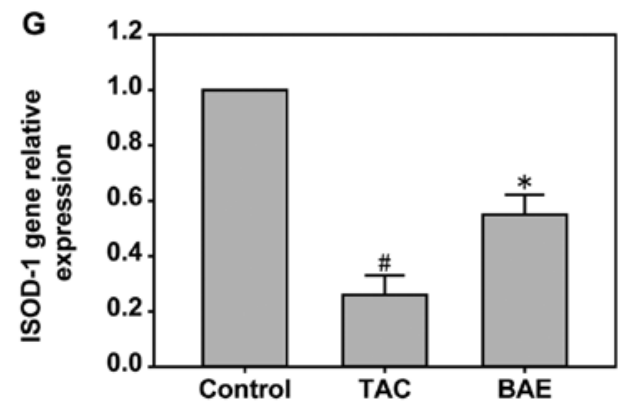

Figure 3. BAE ameliorates TAC-induced oxidative stress in mice. Heart tissues were isolated and the protein and mRNA expression levels of MDA5, IREa and SOD-1 were detected by western blotting and reverse transcription-quantitative PCR, respectively. (A) Representative blot images. (B-D) Quantification of western blotting results. (E-G) mRNA expression levels. All experiments were repeated at least three times. Data are presented as mean \pm standard error of the mean. ${ }^{\#} \mathrm{P}<0.05$ vs. control group; ${ }^{*} \mathrm{P}<0.05$ vs. TAC group. BAE, blueberry anthocyanin-enriched extract; TAC, transverse aortic constriction; MDA5, melanoma differentiation-associated protein 5; IRE $\alpha$, inositol-requiring enzyme $\alpha$; SOD-1, superoxide dismutase-1.

associated with cardiovascular disease $(10,12)$, cancer $(13,36)$ liver diseases $(37)$, preeclampsia $(38,39)$ and ischemic and reperfusion injury (40). The present study demonstrated that TAC-induced a significant increase in ADMA concentration and a decrease in DDAH1 expression and NO production, which were ameliorated by BAE treatment. Previous studies have demonstrated that dietary blueberries have vascular beneficial effects by activating multiple targets including eNOS/NO/cyclic guanosine monophosphate (cGMP), redox and inflammatory signaling pathways (41-43). The production of NO by eNOS involves multiple steps and can be activated in different ways (44). In addition to vasodilation, NO exerts many beneficial vascular effects through anti-inflammation, antiplatelet, antiproliferation and antimigration activity (45). A randomized, double-blinded, placebo-controlled clinical trial study found that daily blueberry supplement reduces blood pressure and arterial stiffness, which may be related to increase NO production (46). In addition, pterostilbene, an active constituent of blueberries, causes eNOS phosphorylation and subsequent NO production through activation of the PI3K/Akt pathway (47); this blueberry supplement may change the intestinal microbiota and the anti-hypertensive effect of blueberries may be regulated by the NO-dependent pathway (48). Collectively, these studies suggest that the vascular effects of blueberries could occur via eNOS/NO/cGMP signaling, but the direct effect of 
A
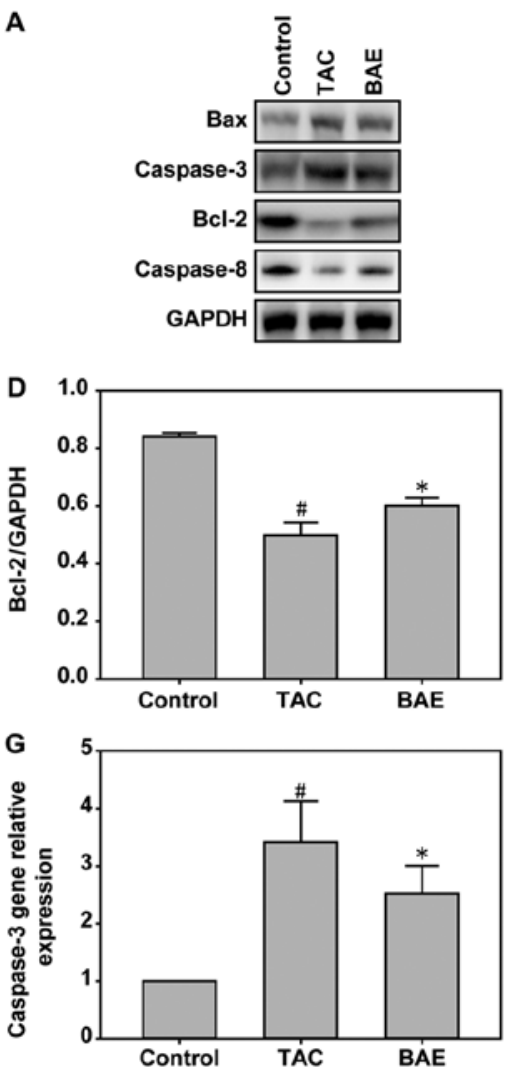

B

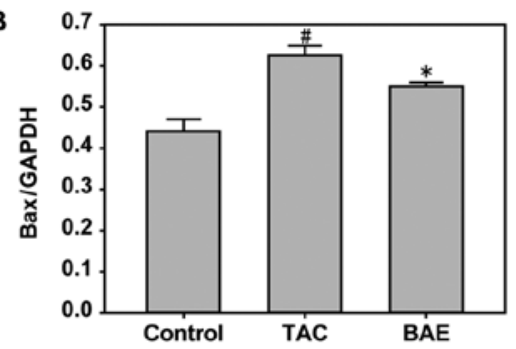

E
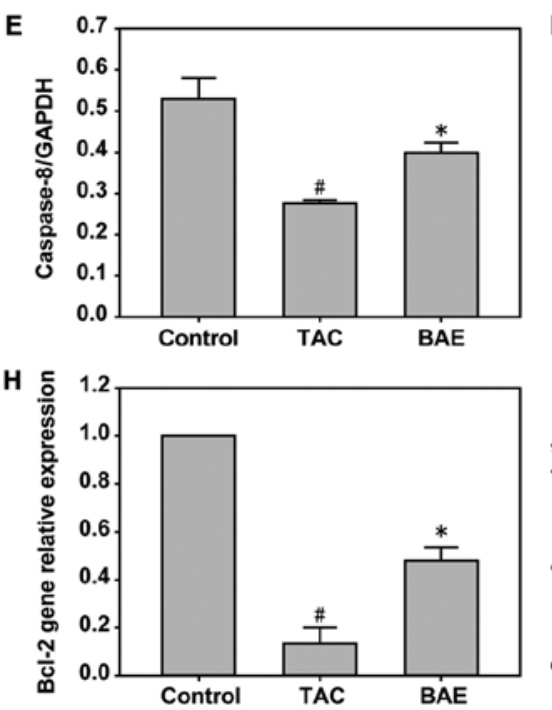

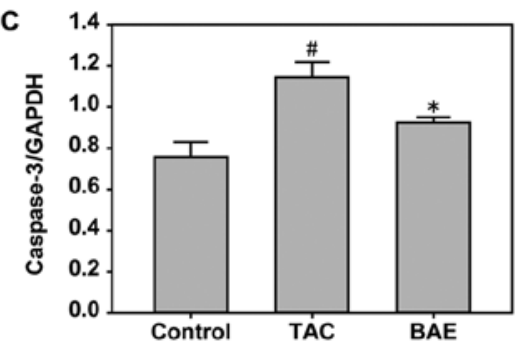

$\mathbf{F}$
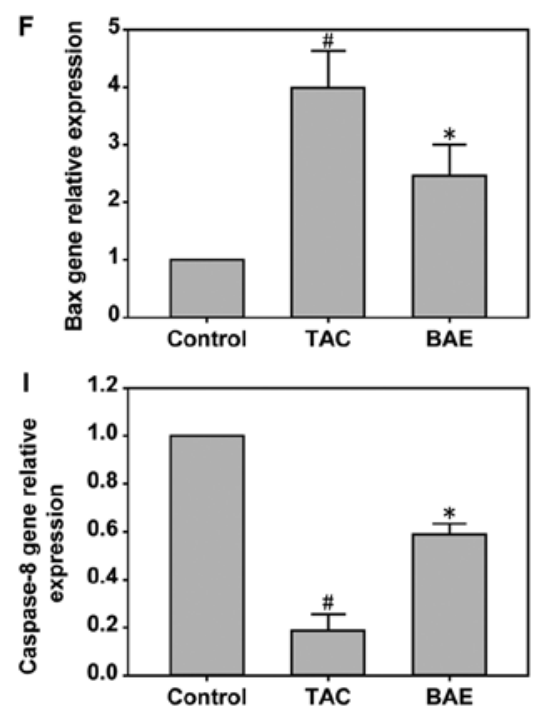

Figure 4. BAE ameliorates TAC-induced heart tissue apoptosis in mice. The protein and mRNA expression levels of Bax, Caspase-3, Bcl-2 and Caspase-8 were detected in heart tissues by western blotting reverse transcription-quantitative PCR, respectively. (A) Representative blot images. (B-E) Quantification of western blotting results. (F-I) mRNA expression levels. All experiments were repeated at least three times. Data are presented as mean \pm standard error of the mean. ${ }^{\#} \mathrm{P}<0.05$ vs. control group; ${ }^{*} \mathrm{P}<0.05$ vs. TAC group. BAE, blueberry anthocyanin-enriched extract; TAC, transverse aortic constriction.

A

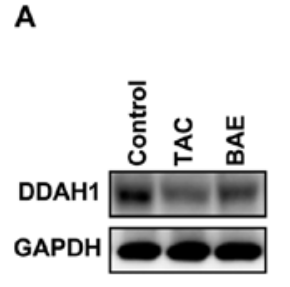

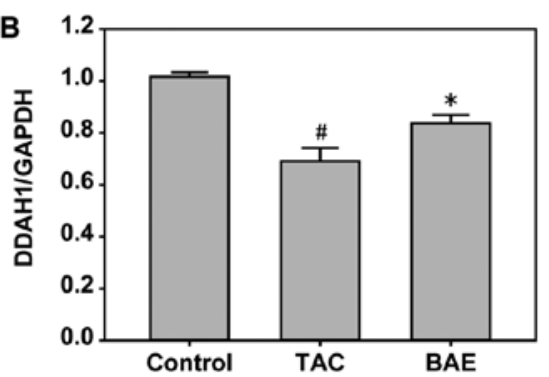
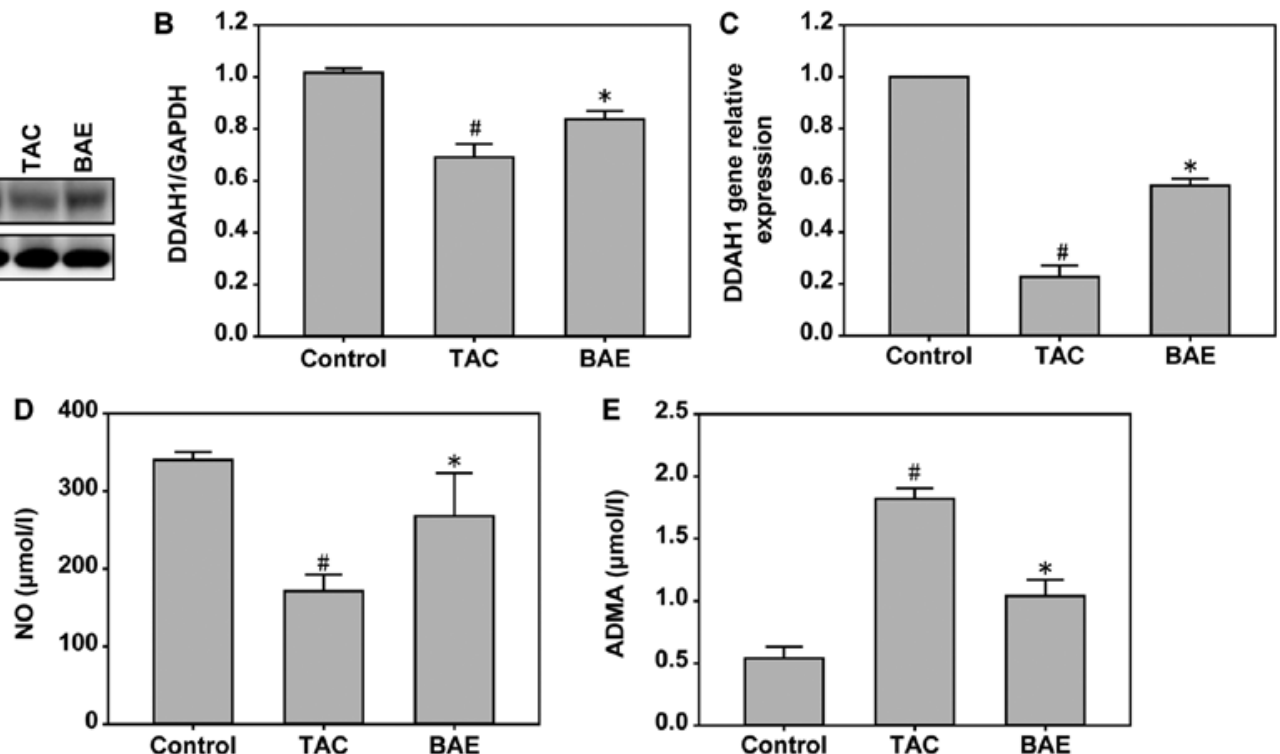

Figure 5. BAE ameliorates TAC-induced myocardial dysfunction through DDAH1/ADMA/NO signaling in mice. (A) The protein expression levels of DDAH1 in heart tissues were detected by western blotting. Representative blot images and (B) quantification are shown. (C) mRNA expression levels of DDAH1 were measured by reverse transcription-quantitative PCR. (D) ADMA concentration and (E) NO production were detected by ELISA. All experiments were repeated at least three times. Data are presented as mean \pm standard error of the mean. ${ }^{~} \mathrm{P}<0.05$ vs. control group; ${ }^{*} \mathrm{P}<0.05$ vs. TAC group. BAE, blueberry anthocyanin-enriched extract; TAC, transverse aortic constriction; DDAH1, dimethylarginine dimethylaminohydrolase 1; ADMA, asymmetric dimethylarginine; $\mathrm{NO}$, nitric oxide.

blueberry anthocyanins on eNOS activation remains to be elucidated.
In conclusion, the data form the present study suggested that BAE exerted potential beneficial vascular effects against 
TAC-induced LV inflammation, oxidative stress and apoptosis, by decreasing ADMA concentration and by elevating DDAH1 expression and NO production. BAE treatment ameliorated the TAC-induced myocardial dysfunction, oxidative stress and inflammatory response and apoptosis, potentially via the DDAH1/ADMA/NO signaling pathway.

\section{Acknowledgements}

Not applicable.

\section{Funding}

No funding was received.

\section{Availability of data and materials}

All data generated or analyzed during the present study are included in this published article.

\section{Authors' contributions}

WW, QM, TL and JFZ performed the experiments, analyzed and interpreted data. WH and JCZ made substantial contributions to the conception and design of the present study, and wrote the manuscript. All authors read and approved the final manuscript.

\section{Ethics approval and consent to participate}

All animal experiments were conducted in compliance with the Guide for the Care and Use of Laboratory Animals published by the National Institutes of Health, and were approved by the Animal Ethics Committee of the People' Hospital of Weifang City (Shandong, China).

\section{Patient consent for publication}

Not applicable.

\section{Competing interests}

The authors declare that they have no competing interests.

\section{References}

1. Muller OJ, Heckmann MB, Ding L, Rapti K, Rangrez AY, Gerken T, Christiansen N, Rennefahrt UEE, Witt H, González Maldonado S, et al: Comprehensive plasma and tissue profiling reveals systemic metabolic alterations in cardiac hypertrophy and failure. Cardiovasc Res 115: 1296-1305, 2019.

2. Quast C, Alter C, Ding Z, Borg N and Schrader J: Adenosine formed by CD73 on $\mathrm{T}$ cells inhibits cardiac inflammation and fibrosis and preserves contractile function in transverse aortic constriction-induced heart failure. Circ Heart Fail 10: pii: e003346, 2017.

3. Shang L, Weng X, Wang D, Yue W, Mernaugh R, Amarnath V, Weir EK, Dudley SC, Xu Y, Hou M and Chen Y: Isolevuglandin scavenger attenuates pressure overload-induced cardiac oxidative stress, cardiac hypertrophy, heart failure and lung remodeling. Free Radic Biol Med 141: 291-298, 2019.

4. Chen Y, Luo HQ, Sun LL, Xu MT, Yu J, Liu LL, Zhang JY, Wang YQ, Wang HX, Bao XF and Meng GL: Dihydromyricetin attenuates myocardial hypertrophy induced by transverse aortic constriction via oxidative stress inhibition and SIRT3 pathway enhancement. Int J Mol Sci 19: pii: E2592, 2018
5. Feng J, Li S and Chen $\mathrm{H}$ : Tanshinone IIA inhibits myocardial remodeling induced by pressure overload via suppressing oxidative stress and inflammation: Possible role of silent information regulator 1. Eur J Pharmacol 791: 632-639, 2016.

6. Gröschel C, Sasse A, Monecke S, Röhrborn C, Elsner L, Didié M, Reupke V, Bunt G, Lichtman AH, Toischer K, et al: $\mathrm{CD}^{+}-\mathrm{T}$ cells with specificity for a model antigen in cardiomyocytes can become activated after transverse aortic constriction but do not accelerate progression to heart failure. Front Immunol 9: 2665, 2018.

7. Wieczór AM, Wieczór R, Kulwas A and Rość D: Asymmetric dimethylarginine and angiogenesis: Biological significance. Int Angiol 37: 431-436, 2018.

8. Dovinová I, Hrabárová E, Jansen E, Kvandová M, Majzúnová M, Berenyiová A and Barančík M: ADMA, homocysteine and redox status improvement affected by 7-nitroindazole in spontaneously hypertensive rats. Biomed Pharmacother 106: 1478-1483, 2018

9. Zarezadeh M, Saedisomeolia A, Khorshidi M, Kord Varkane H, Makhdoomi Arzati M, Abdollahi M, Yekaninejad MS, Hashemi R, Effatpanah M and Mohammadzadeh Honarvar N: Asymmetric dimethylarginine and soluble inter-cellular adhesion molecule-1 serum levels alteration following ginger supplementation in patients with type 2 diabetes: A randomized double-blind, placebo-controlled clinical trial. J Complement Integr Med 16, 2018

10. Liu X, Xu X, Shang R and Chen Y: Asymmetric dimethylarginine (ADMA) as an important risk factor for the increased cardiovascular diseases and heart failure in chronic kidney disease. Nitric Oxide 78: 113-120, 2018.

11. Kirkby NS, Tesfai A, Ahmetaj-Shala B, Gashaw HH, Sampaio W, Etelvino G, Leão NM, Santos RA and Mitchell JA: Ibuprofen arginate retains eNOS substrate activity and reverses endothelial dysfunction: Implications for the COX-2/ADMA axis. FASEB J 30: 4172-4179, 2016.

12. Amir M, Hassanein SI, Abdel Rahman MF and Gad MZ: AGXT2 and DDAH-1 genetic variants are highly correlated with serum ADMA and SDMA levels and with incidence of coronary artery disease in Egyptians. Mol Biol Rep 45: 2411-2419, 2018.

13. Reddy KRK, Dasari C, Duscharla D, Supriya B, Ram NS, Surekha MV, Kumar JM and Ummanni R: Dimethylarginine dimethylaminohydrolase-1 (DDAH1) is frequently upregulated in prostate cancer, and its overexpression conveys tumor growth and angiogenesis by metabolizing asymmetric dimethylarginine (ADMA). Angiogenesis 21: 79-94, 2018.

14. Liu YE, Tong CC, Zhang YB, Cong PF, Shi XY, Liu Y, Shi L, Tong Z, Jin HX and Hou MX: Chitosan oligosaccharide ameliorates acute lung injury induced by blast injury through the DDAH1/ADMA pathway. PLoS One 13: e0192135, 2018.

15. Xu X, Zhang P, Kwak D, Fassett J, Yue W, Atzler D, Hu X, Liu X, Wang H, Lu Z, et al: Cardiomyocyte dimethylarginine dimethylaminohydrolase-1 (DDAH1) plays an important role in attenuating ventricular hypertrophy and dysfunction. Basic Res Cardiol 112: 55, 2017.

16. Li D, Wang P, Luo Y, Zhao M and Chen F: Health benefits of anthocyanins and molecular mechanisms: Update from recent decade. Crit Rev Food Sci Nutr 57: 1729-1741, 2017.

17. Wu X, Beecher GR, Holden JM, Hay towitz DB, Gebhardt SE and Prior RL: Concentrations of anthocyanins in common foods in the United States and estimation of normal consumption. J Agric Food Chem 54: 4069-4075, 2006.

18. Wang Y, Lin J, Tian J, Si X, Jiao X, Zhang W, Gong E and Li B: Blueberry malvidin-3-galactoside suppresses hepatocellular carcinoma by regulating apoptosis, proliferation, and metastasis pathways in vivo and in vitro. J Agric Food Chem 67: 625-636, 2019.

19. deAlmeida AC, van Oort RJ and Wehrens XH: Transverse aortic constriction in mice. J Vis Exp: pii: 1729, 2010.

20. Hampton C, Rosa R, Campbell B, Kennan R, Gichuru L, Ping X, Shen X, Small K, Madwed J and Lynch JJ: Early echocardiographic predictors of outcomes in the mouse transverse aortic constriction heart failure model. J Pharmacol Toxicol Methods 84: 93-101, 2017.

21. Livak KJ and Schmittgen TD: Analysis of relative gene expression data using real-time quantitative PCR and the 2(-Delta Delta C(T)) method. Methods 25: 402-408, 2001.

22. Tong C, Liu Y, Zhang Y, Cong P, Shi X, Liu Y, Shi Hongxu Jin L and Hou M: Shock waves increase pulmonary vascular leakage, inflammation, oxidative stress, and apoptosis in a mouse model. Exp Biol Med (Maywood) 243: 934-944, 2018. 
23. Park KH and Park WJ: Endothelial dysfunction: Clinical implications in cardiovascular disease and therapeutic approaches. J Korean Med Sci 30: 1213-1225, 2015.

24. Liu Y, Tan D, Shi L, Liu X, Zhang Y, Tong C, Song D and Hou M: Blueberry anthocyanins-enriched extracts attenuate cyclophosphamide-induced cardiac injury. PLoS One 10: e0127813, 2015.

25. Basu A, Du M, Leyva MJ, Sanchez K, Betts NM, Wu M Aston CE and Lyons TJ: Blueberries decrease cardiovascular risk factors in obese men and women with metabolic syndrome. J Nutr 140: 1582-1587, 2010.

26. Rodriguez-Mateos A, Ishisaka A, Mawatari K, Vidal-Diez A, Spencer JP and Terao J: Blueberry intervention improves vascular reactivity and lowers blood pressure in high-fat-, high-cholesterol-fed rats. Br J Nutr 109: 1746-1754, 2013.

27. Paneni F, Beckman JA, Creager MA and Cosentino F: Diabetes and vascular disease: Pathophysiology, clinical consequences, and medical therapy: Part I. Eur Heart J 34: 2436-2443, 2013.

28. Elks CM, Reed SD, Mariappan N, Shukitt-Hale B, Joseph JA, Ingram DK and Francis J: A blueberry-enriched diet attenuates nephropathy in a rat model of hypertension via reduction in oxidative stress. PLoS One 6: e24028, 2011.

29. Huang WY, Wu H, Li DJ, Song JF, Xiao YD, Liu CQ, Zhou JZ and Sui ZQ: Protective effects of blueberry anthocyanins against $\mathrm{H} 2 \mathrm{O} 2$-induced oxidative injuries in human retinal pigment epithelial cells. J Agric Food Chem 66: 1638-1648, 2018.

30. Song Y, Huang L and Yu J: Effects of blueberry anthocyanins on retinal oxidative stress and inflammation in diabetes through Nrf2/HO-1 signaling. J Neuroimmunol 301: 1-6, 2016.

31. Vendrame S, Daugherty A, Kristo AS, Riso P and KlimisZacas D: Wild blueberry (Vaccinium angustifolium) consumption improves inflammatory status in the obese Zucker rat model of the metabolic syndrome. J Nutr Biochem 24: 1508-1512, 2013.

32. Xie C, Kang J, Ferguson ME, Nagarajan S, Badger TM and $\mathrm{Wu} \mathrm{X}$ : Blueberries reduce pro-inflammatory cytokine TNF- $\alpha$ and IL- 6 production in mouse macrophages by inhibiting NF- $\kappa B$ activation and the MAPK pathway. Mol Nutr Food Res 55: 1587-1591, 2011.

33. Sun J, Wu Y, Long C, He P, Gu J, Yang L, Liang Y and Wang Y: Anthocyanins isolated from blueberry ameliorates $\mathrm{CCl}_{4}$ induced liver fibrosis by modulation of oxidative stress, inflammation and stellate cell activation in mice. Food Chem Toxicol 120: 491-499, 2018.

34. Wu T, Gao Y, Guo X, Zhang M and Gong L: Blackberry and blueberry anthocyanin supplementation counteract high-fat-diet-induced obesity by alleviating oxidative stress and inflammation and accelerating energy expenditure. Oxid Med Cell Longev 2018: 4051232, 2018.

35. Baba AB, Nivetha R, Chattopadhyay I and Nagini S: Blueberry and malvidin inhibit cell cycle progression and induce mitochondrial-mediated apoptosis by abrogating the JAK/STAT-3 signalling pathway. Food Chem Toxicol 109: 534-543, 2017.

36. Ye J, Xu J, Li Y, Huang Q, Huang J, Wang J, Zhong W, Lin X, Chen W and Lin X: DDAH1 mediates gastric cancer cell invasion and metastasis via Wnt/ $\beta$-catenin signaling pathway. Mol Oncol 11: 1208-1224, 2017.
37. Li T, Feng R, Zhao C, Wang Y, Wang J, Liu S, Cao J, Wang H, Wang T, Guo Y and Lu Z: Dimethylarginine dimethylaminohydrolase 1 protects against high-fat diet-induced hepatic steatosis and insulin resistance in mice. Antioxid Redox Signal 26: 598-609, 2017.

38. Akbar F, Heinonen S, Pirskanen M, Uimari P, Tuomainen TP and Salonen JT: Haplotypic association of DDAH1 with susceptibility to pre-eclampsia. Mol Hum Reprod 11: 73-77, 2005.

39. Powers R and Gefter J: OS075. Endothelial-dependent vascular function is significantly impaired in obesity and restored by overexpression of DDAH1: Evidence for the role of ADMA. Pregnancy Hypertens 2: 218, 2012.

40. Trocha M, Nowak B, Merwid-Lad A, Szuba A, Dzięgiel P, Pieśniewska M, Gomułkiewicz A, Wiśniewski J, Piasecki T, Gziut M, et al: The impact of sitagliptin, inhibitor of dipeptidyl peptidase-4 (DPP-4), on the ADMA-DDAH-NO pathway in ischemic and reperfused rat livers. Adv Clin Exp Med 27: 1483-1490, 2018

41. Gielis JF, Lin JY, Wingler K, Van Schil PE, Schmidt HH and Moens AL: Pathogenetic role of eNOS uncoupling in cardiopulmonary disorders. Free Radic Biol Med 50: 765-776, 2011.

42. Bharat D, Cavalcanti RRM, Petersen C, Begaye N, Cutler BR, Costa MMA, Ramos RKLG, Ferreira MR, Li Y, Bharath LP, et al: Blueberry metabolites attenuate lipotoxicity-induced endothelial dysfunction. Mol Nutr Food Res 62, 2018.

43. Shi D, Xu M, Ren M, Pan E, Luo C, Zhang W and Tang Q: Immunomodulatory effect of flavonoids of blueberry (Vaccinium corymbosum L.) leaves via the NF- $\mathrm{B}$ signal pathway in LPS-stimulated RAW 264.7 cells. J Immunol Res 2017: 5476903 , 2017.

44. Quillon A, Fromy B and Debret R: Endothelium microenvironment sensing leading to nitric oxide mediated vasodilation: A review of nervous and biomechanical signals. Nitric Oxide 45: 20-26, 2015.

45. Su JB: Vascular endothelial dysfunction and pharmacological treatment. World J Cardiol 7: 719-741, 2015.

46. Johnson SA, Figueroa A, Navaei N, Wong A, Kalfon R, Ormsbee LT, Feresin RG, Elam ML, Hooshmand S, Payton ME and Arjmandi BH: Daily blueberry consumption improves blood pressure and arterial stiffness in postmenopausal women with pre- and stage 1-hypertension: A randomized, double-blind, placebo-controlled clinical trial. J Acad Nutr Diet 115: 369-377, 2015.

47. Park SH, Jeong SO, Chung HT and Pae HO: Pterostilbene, an active constituent of blueberries, stimulates nitric oxide production via activation of endothelial nitric oxide synthase in human umbilical vein endothelial cells. Plant Foods Hum Nutr 70: 263-268, 2015.

48. Ahrén IL, Xu J, Önning G, Olsson C, Ahrné S and Molin G: Antihypertensive activity of blueberries fermented by Lactobacillus plantarum DSM 15313 and effects on the gut microbiota in healthy rats. Clin Nutr 34: 719-726, 2015. 